Irish Math. Soc. Bulletin

Number 76, Winter 2015, 35-36

ISSN 0791-5578

\title{
PRECONDITIONING TECHNIQUES FOR SINGULARLY PERTURBED DIFFERENTIAL EQUATIONS
}

\author{
THÁI ANH NHAN
}

This is an abstract of the $\mathrm{PhD}$ thesis Preconditioning techniques for singularly perturbed differential equations written by Thái Anh Nhan, under the supervision of Dr Niall Madden, at the School of Mathematics, Statistics, and Applied Mathematics, National University of Ireland, Galway and submitted in July 2015.

This dissertation is concerned with the numerical solution of linear systems arising from finite difference and finite element discretizations of singularly perturbed reaction-diffusion problems. Such linear systems present several difficulties that make computing accurate solutions efficiently a nontrivial challenge for both direct and iterative solvers.

The poor performance of direct solvers, such as Cholesky factorization, is due to the presence of subnormal floating point numbers in the factors. This thesis provides a careful analysis of this phenomenon by giving a concrete formula for the magnitude of the fill-in entries in the Cholesky factors in terms of the perturbation parameter, $\varepsilon$, and the discretization parameter, $N$. It shows that, away from the main diagonal, the magnitude of fill-in entries decreases exponentially. Furthermore, with our analysis, the location of corresponding fill-in entries associated with some given magnitude can also be determined. This can be used to predict the number and location of subnormals in the factors.

Since direct solvers scale badly with $\varepsilon$, one must use iterative solvers. However, the application of finite difference and finite element discretizations on layer-adapted meshes results in ill-conditioned

2010 Mathematics Subject Classification. 65F10, 65N06, 65N22.

Key words and phrases. Shishkin mesh, singularly perturbed, preconditioning, conjugate gradients.

Received on 14-12-2015.

Support of the Irish Research Council (grant number RS/2011/179) is gratefully acknowledged. 
linear systems. The use of suitable preconditioners is essential. In this thesis we analyze several preconditioning techniques. They include the diagonal and incomplete Cholesky preconditioners for finite difference discretized systems, and a specially designed boundary layer preconditioner for a finite element discretized system. The study of the diagonal and incomplete Cholesky preconditioners focuses on the simplicity and robustness of these techniques; while that of the boundary layer preconditioner is concerned with optimality.

Finally, a novel contribution of this thesis is a pointwise uniform convergence proof for one-dimensional singularly perturbed problems. The central idea of the proof is based on the the preconditioning of the discrete system.

School of Mathematics, Statistics and Applied Mathematics, National University of Ireland, Galway

E-mail address, T.A. Nhan: A.Nhan1@NUIGalway.ie 